\title{
Predicting FTSE 100 Close Price Using Hybrid Model
}

\author{
Bashar Al-hnaity, \\ Department of Electronic and \\ Computer Engineering, \\ Brunel University London, UK, \\ E-mail: Bashar.Al- \\ hnaity@brunel.ac.uk
}

\author{
Maysam Abbod, \\ Department of Electronic and \\ Computer Engineering, \\ Brunel University London, UK, \\ E-mail: Maysam.Abbod \\ @brunel.ac.uk
}

\author{
Maher Alar'raj, \\ Department of Electronic and \\ Computer Engineering, \\ Brunel University London, UK, \\ E-mail: Maher.Alar'raj \\ @brunel.ac.uk
}

\begin{abstract}
Prediction financial time series (stock index price) is the most challenging task. Support vector regression (SVR), Support vector machine (SVM) and back propagation neural network (BPNN) are the most popular data mining techniques in prediction financial time series. In this paper a hybrid combination model is introduced to combine the three models and to be most beneficial of them all. Quantization factor is used in this paper for the first time to improve the single SVM and SVR prediction output. And also genetic algorithm (GA) used to determine the weights of the proposed model. FTSE100 daily index closing price is used to evaluate the proposed model performance. The proposed hybrid model numerical results shows the outperform result over all other single model and the traditional simple average combiner.
\end{abstract}

Keywords- genetic algorithm; support vector regression; styling; support vector machine; Back propagation neural network; hybrid model; FTSE 100 stock index.

\section{INTRODUCTION}

In finance domain, stock price prediction is considered as an important topic, where in recent years many researchers have paid a considerable attention. Stock price characteristics are high volatility, complexity, dynamics, and turbulence and therefore stock price prediction is considered as a challenging task. Prediction the stock price has recorded many attempts using various methodologies such as fundamental methods, technical method and traditional time series methods. In fundamental methods the corporation financial information are used to predict /forecast the supply, demand, profit, industry strength, management abilities and different intrinsic matters affecting the market value and growth potential of a stock[1].

Corporations' financial statements, interim reports, historical financial trends and any forecasts concerning future growth, sales, and profits are included in fundamental, where investors believe that these points should rule the selection processes of stocks and timing of sales. On the other hand technical method analysing recent and historical trends, cycles related to stock price issue and factors beyond that, such as dividend payments, trading volume, index trends, industry group trends and popularity, and volatility of stock of stock [2]. Historical financial information are not the solely information that technical method depend on, but therefore, in order to determine changes in stock and in the market analysts will surmise upon other information such, recent trends in stock price changes, price and earning relationships, the activity volume of particular stock or industry, and other similar indicators [3].

Traditional time series method has different prediction/forecasting techniques to predict stock movements such as, generalized autoregressive conditional heteroskedasticity (GARCH), autoregressive integrated moving average (ARIMA) and multivariate regression. In the last decade computational intelligence / data mining techniques have been introduced in to finance and it has been widely used in prediction stock price [4], [5].

According to [5],[6],[7],[8] neural network ability in learning and generalizing the nonlinear data trends is the main reason of the wide utilization of its application to predict/ forecast stock prices. Moreover, the capability of adapting data patterns and building relationships between input and output can lead to achieve higher accuracy than traditional methods [9], [10]. Support vector machine has great potential and superior performance in practical applications. That can be the reason of the adaptation of the statically learning theory algorithm with the principles of risk minimization structure. Therefore, this unique capability and abilities have drawn the attention of investors as well as researchers to utilize such technique in financial time series prediction/ forecasting. Introducing the insensitivity loss function, regression model, by Vanpanik [11] SVM become called support vector regression SVR. SVR has the ability to solve many problem regarding nonlinear estimation and financial time series prediction so the attention of utilizing such a model has also increased dramatically.

In this paper, the FTSE 100 daily closing price will be predicted. Different data mining techniques will be utilized to capture the non-liner characteristics in a stock price time series. The proposed approach is to combine the support vector machine SVM, support vector regression SVR and BPNN as the weight of these models are determined by the GA. The evaluation of the proposed model performance will be done by using it on the FTSE 100 daily closing price as the illustrative examples. The below examples show that the proposed model outperform all single models and with regard to the possible changes in the data structure, proposed model is shown to be more robust. 
The paper structure is as follow: in section 2 the component models SVM, SVR and BPNN are briefly introduced. Section 3 describes the data pre-processing and the hybrid methodology. The experimental result based on explained above data set presents in section 4 . Finally conclusions and future work are in section 5 .

\section{Methodology}

\section{A. Back propagation neural network (BPNN)}

In modelling time series with non-linear structures, the most commonly used structure is three layers feed-forward back propagation [12]. The weights are determined in back propagation process by building a connections among the nodes based on data training, producing a least-mean-square error measure of the actual or desired and the estimated values from the output of the neural network. The initial values are assigned for the connection weights. In order to update the weights, the error between the predicted and actual output values is back propagated via the network. Minimizing the error the desired and predicted output attempts happened after the procedure of supervised learning [13]. The architecture of this network contains a hidden layer of neurons with non-liner transfer function and an output layer of neurons with non-liner transfer function and an output layer of neurons with liner transfer functions. The below figure illustrate the architecture of a back propagation network, where $x_{j}(j=1,2, \ldots, n)$ represent the input variables; $z_{i}(i=1,2, \ldots, m)$ represent the outputs of neurons in the hidden layer; and $y_{t}(t=1,2, \ldots, l)$ represent the outputs of the neural network [14].

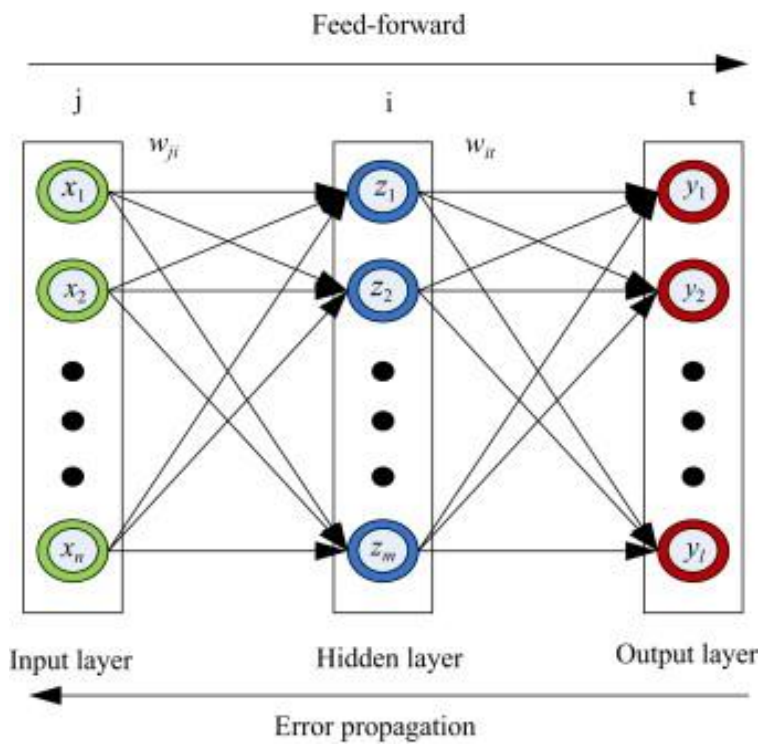

Figure 1. Architecture of feed forward back propagation neural network [14]

In theory neural network has the ability to simulate any kind of data pattern given a sufficient training. Training the neural network will determine the perfect weight to achieve the correct outputs. The following steps illustrate the training process of updating the weights values [15]. First stage is hidden layers: the bellow function explains how the outputs of all neurons in the hidden layer are calculated:

$$
\begin{aligned}
& n e t_{i}=\sum_{j=0}^{n} w_{j i} x_{j} v i=1,2, \ldots, m \\
& z_{i}=f_{H}\left(\text { net }_{i}\right) i=1,2, \ldots ., m
\end{aligned}
$$

where $n e t_{i}$ is the activation value of the ith node, $z_{i}$ is the output of the hidden layer, and $f_{H}$ is called the activation function of a node, in the most cases a sigmoid function as bellow:

$$
f_{H(x)=\frac{1}{1+\exp (-x)}}
$$

Second stage the output: The outputs of all neurons in the output layer are given as bellow function:

$$
y_{t}=f_{t}\left(\sum_{i=0}^{m} w_{i t} z_{i}\right) \quad t=1,2, \ldots, l
$$

where $f_{t}(t=1,2, \ldots, l)$ is the activation function, usually a line function. The weights assigned with random values initially, and are modified by the delta rule according to the learning samples traditionally.

The topology in this study determine by a set of trial and error conducted to choose the best number of neurons experiments, different range of 20 to 5 neurons in hidden layer two layer feed forward back propagation network were tried. The model stopped training after reaching the pre-determined number of epochs. The ideal topology was selected based on the lowest Mean Square Error.

\section{B. Support vector machine (SVM)}

SVM theory was developed by Vladimir Vapnik in 1995. It is considered as one of the most important breakthroughs in machine learning field and can be applied in classification and regression [16]. In modelling the SVM, the main goal is to select the optimal hyperplane in high dimensional space ensuring that the upper bound of generalization error is minimal. SVM can only directly deal with linear samples but mapping the original space into a higher dimensional space can make the analysis of nonlinear sample possible [17], [18]. For example if the data point $(x i, y i)$, was given randomly and independently generated from an unknown function, the approximate function form by SVM is as follow:

$g(x)=w \varnothing(x)+b . \varnothing(x)$ Is the feature and nonlinear mapped from the input space $x$. $w$ and $b$ are both coefficients and can be estimated by minimizing the regularized risk function.

$$
\begin{gathered}
R(C)=C \frac{1}{N} \sum_{i=1}^{N} L\left(d_{i}, y_{i}\right)+\frac{1}{2}\|w\|^{2} \\
L(d, y)=\left\{\begin{array}{cc}
|d-y|-\varepsilon & |d-y| \geq \varepsilon, \\
0 & \text { other, }
\end{array}\right.
\end{gathered}
$$

In the above function both $\mathrm{C}$ and $\varepsilon$ are prescribed parameters. $\mathrm{C}$ is called the regularization constant while $\varepsilon$ is referred to as the regularization constant. $L(d, y)$ Is the intensive loss function and the term $c \frac{1}{N} \sum_{i=1}^{N} L\left(d_{i}, y_{i}\right)$ is the empirical error while the $\frac{1}{2}\|w\|^{2}$ indicates the flatness of the function. The trade-off between the empirical risk and flatness of the model is measured by C. Since introducing positive slack variables $\zeta$ and $\zeta^{*}$ the equation number 6 transformed to the following:

$$
R\left(w, \zeta, \zeta^{*}\right)=\frac{1}{2} w w^{T}+C \times\left(\sum_{i=1}^{N}\left(\zeta, \zeta^{*}\right)\right)
$$


Subject to:

$$
\begin{aligned}
& w \emptyset\left(x_{i}\right)+b_{i}-d_{i} \leq \varepsilon+\zeta *_{i} \\
& d_{i}-w \varnothing\left(x_{i}\right)-b_{i} \leq \varepsilon+\zeta_{i} \\
& \zeta_{i}, \zeta_{l} \geq 0
\end{aligned}
$$

The decision function (kernel function) comes up finally after the Lagrange multipliers are introduced and optimality constraints exploited. The below function is the form of kernel function:

$$
f(x)=\sum_{i}^{l}\left(\alpha_{i}-\dot{\alpha}_{i}\right) K\left(x_{i}, x_{j}\right)+b
$$

where $\dot{\alpha}_{i}$ are Lagrange multipliers. The satisfy the equalities $\alpha_{i} \times \dot{\alpha}_{i}=0, \alpha_{i} \geq 0, \dot{\alpha}_{i} \geq 0$. The kernel value is the same with the inner product of two vectors $x_{i}$ and $x_{j}$ in the feature space $\varnothing\left(x_{i}\right)$ and $\varnothing\left(x_{j}\right)$. The most popular kernel function is Radial Basis Function (RBF) it is form in equation (12).

$$
K\left(x_{i}, x_{j}\right)=\exp \left(-\gamma\left\|x_{i}-x_{j}\right\|^{2}\right)
$$

Theoretical background, geometric interpretation, unique solution and mathematical tractability are the main advantages which has made SVM attract researchers and investors interest and be applied to many application in different fields such prediction financial time series.

\section{Support vector regression (SVR)}

As explained above SVM idea is to constructs a hyperplane or set of hyperplanes in a high or infinite dimensional space, which can be used for classification. In the regression problem the same margin concept used in SVM is used. The goal of solving regression problem is to construct a hyperplane that close to as many of the data points as possible. Choosing a hyperplane with small norm is considered as a main objective, while simultaneously minimizing the sum of the distances from the data points to the hyperplane [19].

In case of solving regression problem using SVM, SVM became called support vector regression (SVR) where the aim is to find a function $f$ with parameters $\mathrm{w}$ and $\mathrm{b}$ by minimizing the following regression risk :

$$
R(f)=\frac{1}{2}(x, w)+C \sum_{i=1}^{N} l\left(f\left(x_{i}\right), y_{i}\right)
$$

The above function $\mathrm{C}$ is a trade-off term, the margin in SVM is the first term which is used in measuring VC-dimension [15].

$$
f(x, w, b)=(w, \varnothing(x))+b,
$$

In the function above $\emptyset(x): x \rightarrow \Omega$ is kernel function, mapping $\mathrm{x}$ into in the high dimensional space.

SVR and as proposed by [19]. The $\varepsilon-$ insensitive loss function is used as follows:

$$
l(y, f(x))=\left\{\begin{array}{c}
0, \text { if }|y-f(x)|<\varepsilon \\
|y-f(x)|-\varepsilon, \text { otherwise }
\end{array}\right.
$$

Bellow constrained minimization problem is equivalent previous minimization in equation (13).

$$
\text { Min } y\left(w, b, \xi^{(*)}=\frac{1}{2}(w, w)+C \sum_{i=1}^{N}\left(\xi_{i}+\xi_{i^{*}}\right)\right.
$$

subject to:

$$
y_{i}-\left(\left(w, \phi\left(x_{i}\right)\right)++b\right) \leq \varepsilon+\xi_{i},
$$

$$
\begin{aligned}
& \left(\left(w, \phi\left(x_{i}\right)\right)+b\right)-y_{i} \leq \varepsilon+\xi_{i^{*}}, \\
& \xi_{i}^{*} \geq 0
\end{aligned}
$$

In sample $\left(x_{i}, y_{i}\right)$ the $\xi_{i}$ and $\xi_{i}$ measure the up error and down error. Maximizing the dual function or in other words construct the dual problem of this optimization problem (primal problem) by large method is a standard method to solve the above minimization problem. There are four common kernel functions, in this study radial basis function (RBF) will be used.

\section{THE Framework of Prediction MODELS}

\section{A. Hybrid model}

Combining different prediction techniques has been investigated widely in the literature. In the short range prediction combining the various techniques is more useful according to [20], [21]. [22] Stated in his study that using simple average may work as well as more sophisticated approaches. However, using one model can produce more accurate prediction than any other methods. Simple averages would not be sufficient in such cases [22]. Compared with different prediction models hybrid prediction method is based on a certain linear combination. The assumption for the actual value in period $t$ is $y_{t}(t=1,2, \ldots, m)$ in prediction problem where $\mathrm{m}$ the prediction of different types. If the prediction value in period $\mathrm{t}$ by model $i$ is $f_{i t}(i=1,2, \ldots, m)$, the corresponding prediction error will be $e_{i t}=y_{t}-f_{i t}$. And also the weight vector will be $W=\left[w_{1}, w_{2}, \ldots, w_{m}\right]^{T}$. Then in the hybrid model the predicted value is computed as follows [23], [24]:

$$
\begin{aligned}
& \widehat{y_{t}}=\sum_{i=1}^{m} w_{i} f_{i t}(t=1,2, \ldots, n) \\
& \sum_{i=1}^{m} w_{i}=1
\end{aligned}
$$

Equation (21) can be expressed in another form such:

$$
\hat{Y}=F W
$$

where $\widehat{Y}=\left[\widehat{y_{1}}, \widehat{y_{2}}, \ldots ., \widehat{y_{n}}\right]^{T}, F=\left[f_{i t}\right]_{n \times m}$.

The error for the prediction model can be formed as bellow equation:

$$
\begin{gathered}
e_{t}=y_{t}-\widehat{y_{t}}=\sum_{i=1}^{m} w_{i} y_{t}-\sum_{i=1}^{m} w_{i} f_{i t}= \\
\sum_{i=1}^{m} w_{i}\left(y_{t}-f_{i t}\right)=\sum_{i=1}^{m} w_{i} e_{i t}
\end{gathered}
$$

This study proposed a hybrid model of combining BPNN, SVM and SVR.

$$
\begin{aligned}
\hat{Y}_{\operatorname{combined}_{(t)}=} & w_{1} \hat{Y} S V M_{(t)} \\
& +w_{2} \hat{Y} S V R_{(t)} \quad(t=1,2, \ldots, n)
\end{aligned}
$$

The prediction values in period $t$ $\operatorname{are}_{\hat{Y}}$ combined $_{(t)}, w_{1} \hat{Y} S V M_{(t)}$ and $w_{2} \hat{Y} S V R_{(t)}$ for the hybrid BPNN, SVM and SVR and $w_{i}(i=1,2,3)$ are the assigned weight for BPNN, SVM and SVR models. 
The most important step in developing a hybrid prediction model is to determine the perfect weight for each individual model. Setting the $w_{1}=w_{2}=w_{3}=1 / 3$ is the simplest combination method for the three prediction models. Therefore, in many cases equal weights cannot achieve the best prediction result. Thus, this paper utilizes GA to determine the optimal weight for each prediction model. Figure 2 illustrate the architecture of the hybrid prediction model. Solving the optimization problems is one of main issue that genetic algorithm used to solve, in this paper GA utilized to determine a set of optimal weight for the hybrid model.

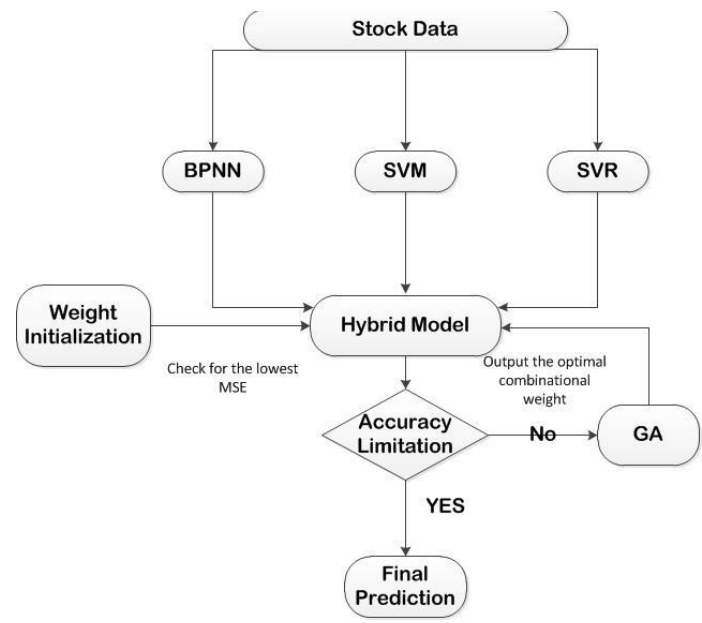

Figure 2. Architecture of hybrid model

\section{B. Data pre-processing}

This paper examined FTSE100 index. The data were collected from London stock exchange market. Closing daily price was used. Data set covers a period of 03/01/1984 to 30/10/2014 (including 8038 points). The data set is divided into two sets, training and testing. The first 7788 data is used as a training data set and the last 250 is used as a testing data set. This study considers one-step-ahead prediction in order to prevent problems associated with cumulative error from previous period for out of sample prediction.

Selection and pre-processing the data are crucial step in any modelling effort, particularly for generalizing the new predictive model. Data set are divided into two sets: for training dataTr $=\left[x_{(m)} \ldots \ldots \ldots x_{m+5}\right]\left[x_{(m+6)}\right]$, where $\mathrm{m}$ is a random number permutation $1<m<N_{T r}, N_{T r}$ is the data size. For testing data $T s=\left[x_{(n)} \ldots \ldots x_{(n+5)}\right]\left[x_{(x+6)}\right]$, where $n$ is $1: N_{T S}, N_{T s}$ is the testing data size.

\section{Determining the parameters in SVM, SVR models.}

This study used SVM and SVR to predict FTSE 100 daily closing price. If the parameter of each model set properly that could improve the prediction output for each model. In both model RBF kernel function has been used, two parameters should be determine $C$ and $\gamma$ in both model. The most common approach to search the best $C$ and $\gamma$ values is grid search approach [20]. Grid research approach uses a validation process in order to achieve the ability to produce good generalizations to decide parameters. The criteria of choosing the optimal $C$ and $\gamma$ parameters is by tried pairs of $C$ and $\gamma$ and the best cross validation prediction of mean square error MSE is chosen.

On the grid and after identifying a better region, on this region more specific grid search can be conducted [25]. Finally after determining these lowest cross validation prediction error parameters $C$ and $\gamma$, choose these parameters to be used in creation the prediction models. As figure 3 illustrate the process of grid search algorithm when building the SVM, SVR prediction model.

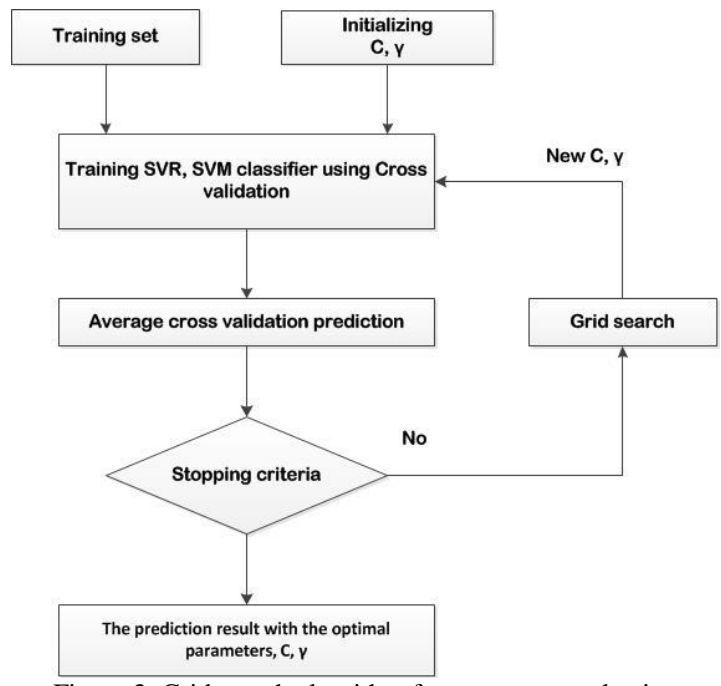

Figure 3. Grid search algorithm for parameters selection

\section{Quantization factor}

This paper adopts a new approach to enhance the prediction output of SVM and SVR model. Quantization factor for the first time introduces to SVM and SVR. In this paper factor has been added the SVM and SVR model. As explained above in the methodology section SVM and SVR the model input is $(x i, y i)$. After adding the optimal factor which were determine by trial and error, conducted to choose the optimal factor from a range of factor between 10-100. The optimal factor was selected based on the lowest mean square error. The below steps illustrate the changing on the input after introduce the quantization factor:

$$
\begin{aligned}
& \text { Xprim }=X_{i} / \text { factor } . \\
& \text { Yprime }=y_{i} / \text { factor } .
\end{aligned}
$$

The model inputs become $\left(\right.$ Xprime $_{i}$, Yprime $\left._{i}\right)$. After applying the above model SVM and SVR and to get the final prediction result the chosen factor multiply with the output of each model as below:

$$
\begin{aligned}
& \text { Xprim_pred }=\text { Xprim } \times \text { factor } . \\
& \text { Yprime } e_{\text {pred }}=\text { Yprime } \times \text { factor } .
\end{aligned}
$$

\section{E. Algorithm implementation}

SVR and SVM packages are available from different resources such: Joachims [26], LibSVM which was proposed by Chih-jen Lin [27] and Steve Gunn Matlab Toolbox. This study will utilize LibSVM. 


\section{F. Prediction result evaluation}

In this paper mean square error (MSE) and root mean square error (RMSE) are used to evaluate the prediction performance for BPNN, SVM, SVR and the hybrid model. The below function are the forms of these measures:

$$
\begin{aligned}
& \mathrm{MSE}=\frac{1}{N} \sum_{t=1}^{N}(x(t)-\hat{x}(t))^{2} \\
& \mathrm{RMSE}=\left[\frac{1}{N} \sum_{t=1}^{N}(x(t)-\hat{x}(t))^{2}\right]^{\frac{1}{2}}
\end{aligned}
$$

\section{EXPERIMENTS AND PREDICTION RESULTS}

\section{A. Data sets}

This paper proposes a hybrid model to predict the index closing price. In order to test the proposed model, FTSE 100 index closing price form London stock market is used. The data set cover the period form 03/01/1984 to 30/10/2014 figure 4 show the time series plot. There are 8038 data points in total. The first 7788 are used as training data set and the remaining 250 are used as a testing data set.

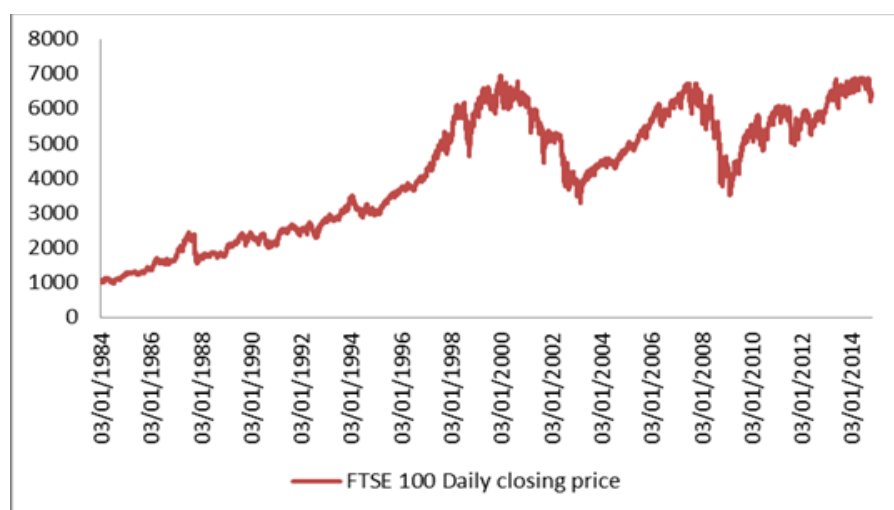

Figure 4. FTSE100 daily closing price

\section{B. Prediction results}

This paper proposes a hybrid method combining three different models SVM, SVR and BPNN to predict FTSE 100 stock index closing price. First step to build the proposed method is by performing the single classical models on the data set individually. Building a BPNN prediction model by determining the architecture as mentioned in the methodology section in BPNN part. BPNN topology has been set by trial and error techniques, two hidden layer feed forward back propagation network with only one hidden layer and a range of 20-5 is the range of neurons. 0.7 is the learning rate which gives the minimum testing error. Figure 5 illustrates the prediction results of BPNN.

To build the SVR and SVM model, the method which is addressed in the methodology section and in the framework prediction model section to determine the parameters of both model and the factor is applied. In both model the parameters and factor are as follow $\mathrm{C}=100, \gamma=0.0003$ and the factor $=17$ with MSE 0.0002. Figure 6 shows the prediction results of SVR model and figure 7 shows the prediction results of SVM model.

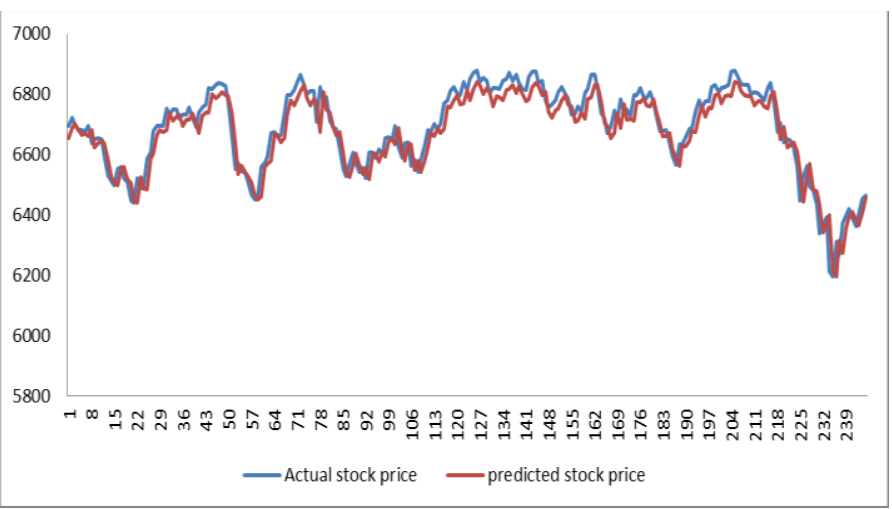

Figure 5. The prediction result of FTSE 100 based on BPNN

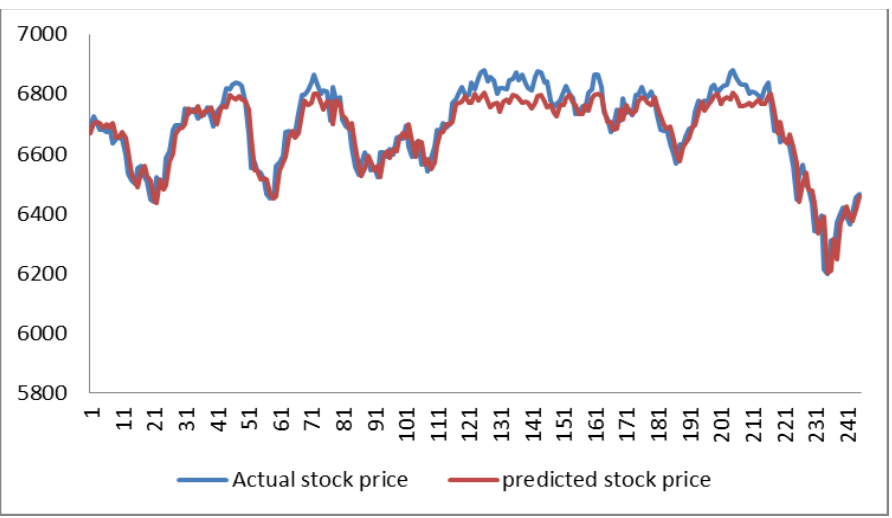

Figure 6. The prediction result of FTSE 100 based on SVR

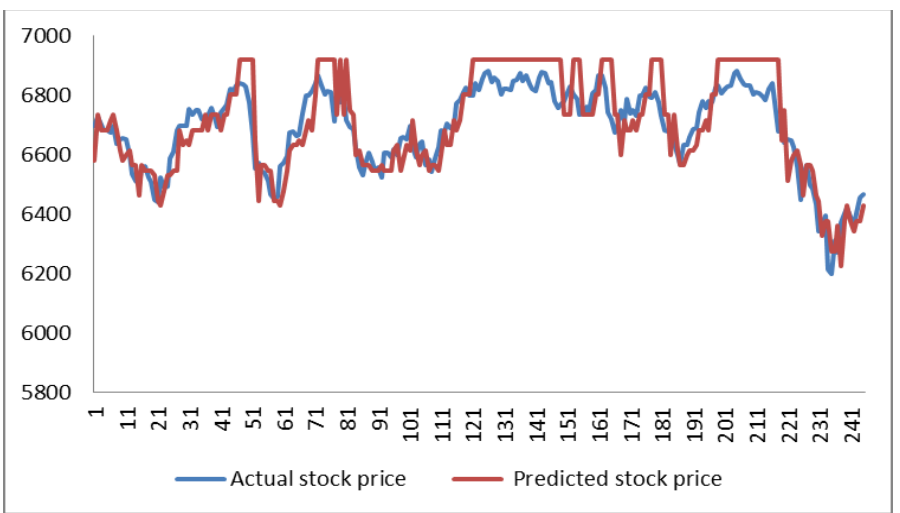

Figure 7. The prediction result of FTSE 100 based on SVM

Figure 8 shows the proposed hybrid model prediction results while figure 9 shows the prediction results from using a basic combiner average.

The prediction performance evaluated in this paper by compare the all five model hybrid proposed model, SVM model, SVR model, BPNN model and the simple average combination model. Table I. Present the two performance measures of MSE and RMSE of the five models. It is obvious that the propose model outperform all models and has much less error than the rest models.

\section{CONCLUSIONS}

This paper examines the predictability of stock index time series whereas the dynamics of such a data in real situations is 
complex and unknown. As a result using single classical model will not produce the accurate prediction result. In this paper a hybrid combination model was introduced combining BPNN, SVM and SVR. FTSE 100 daily closing price was used to evaluate the proposed model performance. The proposed model was compared with different models and as the table I showed that the hybrid model outperforms all other models.

From the result that were achieved in this paper, the proposed hybrid model outperform the other three single models, thus future work should revolve around different hybrid combination models paradigm. Different singles models can be added and the possibility of combining them. Moreover, to test the model robustness further extension of this study can be done by testing different dataset.

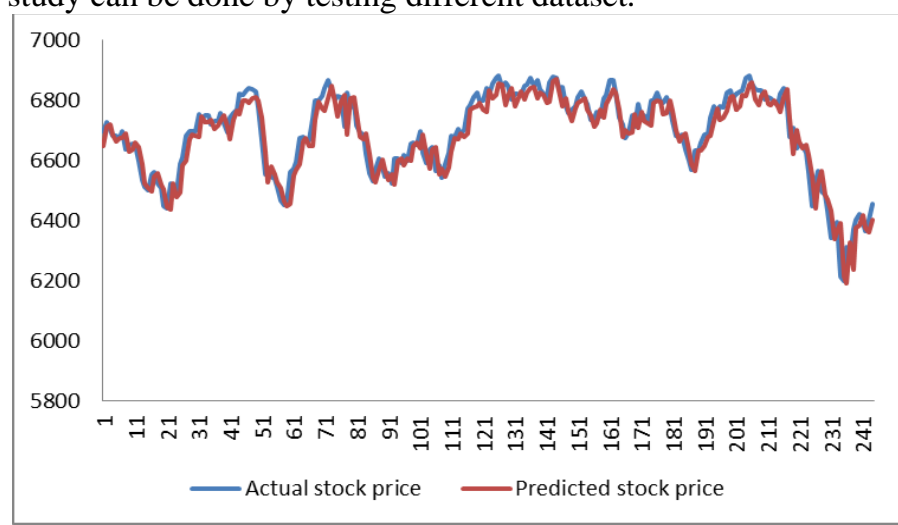

Figure 8 . The prediction result of FTSE 100 based on hybrid proposed model

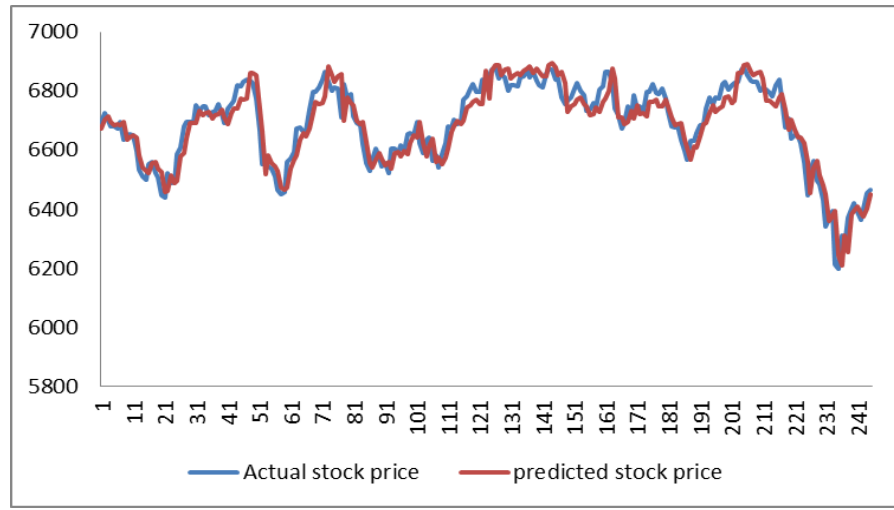

Figure 9. The prediction result of FTSE 100 based on average simple combination

TABLE I. PREDICTION ERROR OF DIFFERENT MODELS

\begin{tabular}{|c|c|c|}
\hline \multirow{2}{*}{ Prediction models } & \multicolumn{2}{|c|}{ Prediction Error } \\
\cline { 2 - 3 } & $\boldsymbol{M S E}$ & $\boldsymbol{R M S E}$ \\
\hline BPNN & 2210.75 & 47.02 \\
\hline SVM & 6532.26 & 80.82 \\
\hline SVR & 2438.87 & 49.38 \\
\hline SA combination model & 2410.96 & 49.10 \\
\hline Hybrid proposed model & $\mathbf{2 0 6 6 . 6 5}$ & $\mathbf{4 5 . 4 6}^{*}$ \\
\hline
\end{tabular}

\section{REFERENCES}

[1] M. C. Thomsett, Mastering Fundamental Analysis. Dearborn Financial Pub., 1998.
[2] T. Bollerslev, "Generalized autoregressive conditional heteroskedasticity," J. Econ., vol. 31, pp. 307-327, 1986.

[3] A. Zellner and F. Palm, "Time series analysis and simultaneous equation econometric models," J. Econ., vol. 2, pp. 17-54, 1974.

[4] A. Adebiyi, C. Ayo, M. Adebiyi and S. Otokiti, "An Improved Stock Price Prediction using Hybrid Market Indicators," African Journal of Computing \& ICT, vol. 5, pp. 124-135, 2012

[5] M. Khashei, M. Bijari and G. A. Raissi Ardali, "Improvement of autoregressive integrated moving average models using fuzzy logic and artificial neural networks (ANNs)," Neurocomputing, vol. 72, pp. 956967, 2009.

[6] M. Khashei and M. Bijari, "An artificial neural network ( p, d, q) model for timeseries forecasting," Expert Syst. Appl., vol. 37, pp. 479489, 2010

[7] E. Hajizadeh, H. D. Ardakani and J. Shahrabi, "Application of data mining techniques in stock markets: A survey," Journal of Economics and International Finance, vol. 2, pp. 109-118, 2010.

[8] C. S. Vui, G. K. Soon, C. K. On, R. Alfred and P. Anthony, "A review of stock market prediction with artificial neural network (ANN)," in Control System, Computing and Engineering (ICCSCE), 2013 IEEE International Conference on, 2013, pp. 477-482.

[9] S. Soni, "Applications of ANNs in stock market prediction: a survey," International Journal of Computer Science \& Engineering Technology, vol. 2, pp. 71-83, 2011.

[10] H. Yu and H. Liu, "Improved stock market prediction by combining support vector machine and empirical mode decomposition," in Computational Intelligence and Design (ISCID), 2012 Fifth International Symposium on, 2012, pp. 531-534.

[11] V. Vapnik, The Nature of Statistical Learning Theory. Springer Science \& Business Media, 2000.

[12] L. A. Diaz-Robles, J. C. Ortega, J. S. Fu, G. D. Reed, J. C. Chow, J. G. Watson and J. A. Moncada-Herrera, "A hybrid ARIMA and artificial neural networks model to forecast particulate matter in urban areas: the case of Temuco, Chile," Atmos. Environ., vol. 42, pp. 8331-8340, 2008.

[13] M. Kubat, Neural Networks: A Comprehensive Foundation by Simon Haykin, Macmillan, 1994, ISBN 0-02-352781-7., 1999.

[14] Y. Wang, "Nonlinear neural network forecasting model for stock index option price: Hybrid GJR-GARCH approach," Expert Syst. Appl., vol. 36, pp. 564-570, 2009.

[15] D. Ö. Faruk, "A hybrid neural network and ARIMA model for water quality time series prediction," Eng Appl Artif Intell, vol. 23, pp. 586594, 2010.

[16] C. Cortes and V. Vapnik, "Support-vector networks," Mach. Learning, vol. 20, pp. 273-297, 1995.

[17] M. Pontil and A. Verri, "Properties of support machines," Neural Comput., vol. 10, pp. 955-974, 1998.

[18] E. Osuna, R. Freund and F. Girosi, "Support vector machines: Training and applications," 1997.

[19] Y. Xia, Y. Liu and Z. Chen, "Support vector regression for prediction of stock trend," in Information Management, Innovation Management and Industrial Engineering (ICIII), 2013 6th International Conference on, 2013, pp. 123-126.

[20] Y. Zhang and L. Wu, "Stock market prediction of S\&P 500 via combination of improved BCO approach and BP neural network," Expert Syst. Appl., vol. 36, pp. 8849-8854, 2009.

[21] J. S. Armstrong, "Combining forecasts: The end of the beginning or the beginning of the end?" Int. J. Forecast., vol. 5, pp. 585-588, 1989.

[22] A. Timmermann, "Forecast combinations," Handbook of Economic Forecasting, vol. 1, pp. 135-196, 2006.

[23] S. Gupta and P. C. Wilton, "Combination of forecasts: An extension," Management Science, vol. 33, pp. 356-372, 1987

[24] C. Christodoulos, C. Michalakelis and D. Varoutas, "Forecasting with limited data: Combining ARIMA and diffusion models," Technological Forecasting and Social Change, vol. 77, pp. 558-565, 2010.

[25] C. Hsu, C. Chang and C. Lin, A Practical Guide to Support Vector Classification, 2003.

[26] T. Joachims, Making Large Scale SVM Learning Practical, 1999

[27] S. Soni, "Applications of ANNs in stock market prediction: a survey," International Journal of Computer Science \& Engineering Technology, vol. 2, pp. 71-83, 2011. 